\title{
Letters
}

\section{On the velocity-dependent fracture toughness of epoxy resins}

In a recent paper [1], Drs Selby and Miller described three methods of determining the fracture toughness $(R)$, or equivalently, the fracture surface energy $(2 \gamma)$ for an epoxy resin. These methods are respectively (a) Berry's method, (b) Irwin-Kies equation and (c) Gurney's irreversible work area method. While the experimental data obtained from tapered double-cantilever beam (TDCB) specimens supported the equivalence of the first two methods (based on crack initiation), they apparently have difficulties in the interpretation of these data using Gurney's method because of the "crack-jumping" nature of the epoxy resin. They have found that the segmental areas give fracture surface energies $(2 \gamma)$ less than that obtained from either Berry's method or Irwin-Kies equation. It would thus appear at first sight that there is a limitation to the usefulness of the Gurney-method for fracture toughness determination for such "crack-jumping" materials. In the present note, we wish to show that such limitations really do not exist once the criteria governing stability in cracking are investigated.

Conditions governing the stability of cracks under constraints of both load- and displacementcontrolled machines are given in [2-4]. In general, the stability criteria may be written as

$$
\begin{aligned}
& \frac{1}{R} \frac{\mathrm{d} R}{\mathrm{~d} a} \geqslant \frac{\mathrm{d}^{2}(u / X)}{\mathrm{d} a^{2}} / \frac{\mathrm{d}}{\mathrm{d} a}\left(\frac{u}{X}\right) \\
& -2 \frac{\mathrm{d}}{\mathrm{d} a}\left(\frac{u}{X}\right) /\left(\frac{u}{X}\right)=\frac{n_{u}}{a}
\end{aligned}
$$

for a displacement-controlled machine (i.e. $\delta u / u \geqslant$ 0 , such as a screw-driven machine) and

$$
\frac{1}{R} \frac{\mathrm{d} R}{\mathrm{~d} a} \geqslant \frac{\mathrm{d}^{2}}{\mathrm{~d} a^{2}}\left(\frac{u}{X}\right) / \frac{\mathrm{d}}{\mathrm{d} a}\left(\frac{u}{X}\right)=\frac{n_{x}}{a}
$$

for a load-controlled machine (i.e. $\delta X / X \geqslant 0$, such as a hydraulic testing machine). $X, u$ and $a$ are, respectively, the load, displacement and crack length. Stability is seen to depend upon two factors, (a) one geometrical (the compliance terms on the right hand side of the inequalities, which 2000 are called the "geometric stability factors" (gsf) for the testpiece under investigation), and (b) one concerning $\mathrm{d} R / \mathrm{d} a$ or $\mathrm{d} R / \mathrm{d} \dot{a}$, i.e. rate dependence in the fracture toughness. If a material displays constant $R$ during cracking, negative values of the gsf promote stable cracking. If, however, the material under test possesses negative $\mathrm{d} R / \mathrm{d} \dot{a}$ characteristics, unstable cracking should be expected uniess testpieces with large negative gsf's are chosen. Methods of promoting stability of cracking have been quite extensively covered in Gurney and Mai [2] . For simple bending type testpiece geometries, stability may be greatly improved by mounting external stabilizers such as adhesive tapes-or rate sensitive devices across the crack path [2]. For moderately unstable materials, cracks can also be stabilized by choosing specimen geometries with sufficiently negative gsf's.

It has previously been reported that "crackjumping" is a characteristic of epoxy polymers [1, 5-7]. Physically, this means that the fracture toughness $(R)$ must be rate or crack speed $(\dot{a})$ dependent such that $\mathrm{d} R / \mathrm{d} \dot{a}$ is negative. This fact is also supported by the authors' own data in that $(2 \gamma)$ or $K_{\text {IC }}$ values decrease with increasing crosshead speed $(\dot{u})$ of the Instron testing machine.

It may be shown [e.g. 3,8] that the relation between $\dot{a}$ and $\dot{u}$ for linear elastic structural systems is given by

$$
\frac{\dot{a}}{\dot{u}}=\frac{1}{t}-\frac{X}{[2 R+u(\mathrm{~d} X / t \mathrm{~d} a)]}
$$

where $t$ is the thickness of the fracture plane.

For the tapered DCB specimen,

$$
\begin{aligned}
\frac{\mathrm{d} X}{\mathrm{~d} a} \approx 0 \quad(R \text { constant }) \\
X \propto \sqrt{ } R(=2 \gamma) ;
\end{aligned}
$$

we have,

$$
\left(\frac{\dot{a}_{1}}{\dot{a}_{2}}\right)=\left(\frac{\dot{u}_{1}}{\dot{u}_{2}}\right)\left(\frac{R_{2}}{R_{1}}\right)\left(\frac{X_{1}}{X_{2}}\right)=\left(\frac{\dot{u}_{1}}{\dot{u}_{2}}\right)\left(\frac{R_{2}}{R_{1}}\right)^{\frac{1}{2}} .
$$

Taking the authors' original data from Table IV in [1], with $\dot{u}_{1}=0.1 \mathrm{~mm} \mathrm{~min}^{-1}, R_{1}=412 \mathrm{~J} \mathrm{~m}^{-2}$; $\dot{u}_{2}=10 \mathrm{~mm} \mathrm{~min}^{-1}$ and $R_{2}=252 \mathrm{~J} \mathrm{~m}^{-2}$; we have $\dot{a}_{1} / \dot{a}_{2} \approx 8 \times 10^{-3}$. This represents roughly a $40 \%$

(c) 1975 Chapman and Hall Ltd. Printed in Great Britain. 


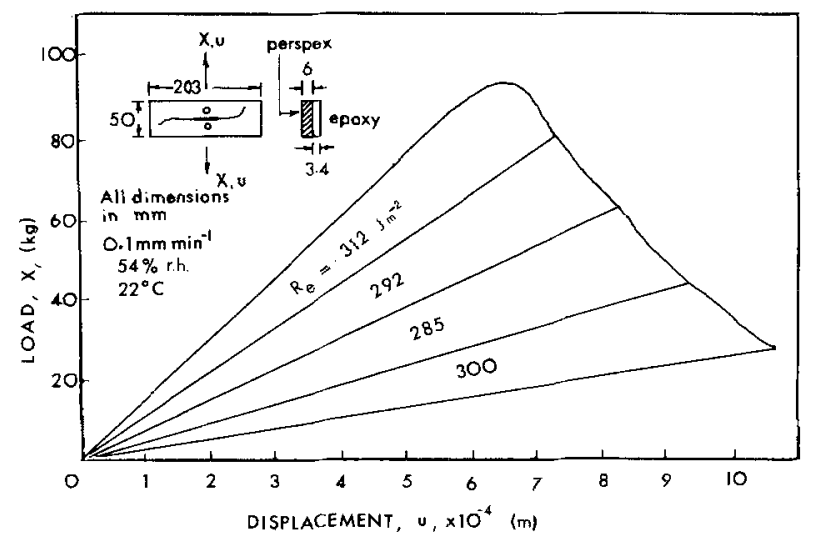

Figure 1 Stabilizing an epoxy resin using a Perspex back plate. reduction in $R$ with an approximately two orders of magnitude increase in crack speed. It is, therefore, clear that testpiece geometries with only moderately favourable gsf's (such as DCB speciments [3], and conventional TDCB specimens $[8,9])$ will not give stable cracking in epoxy resins because $\mathrm{d} R / \mathrm{d} \dot{a}$ is very negative. On top of this, a $\mathrm{d} R / \mathrm{d} a$ negative effect due to crack tip blunting as observed by the authors for epoxy resins with more than 20 parts of DDM will aggravate the already unstable situation.

Attempts to stabilize cracking in an epoxy resin have been tried by Evans and Barr [6]. It is questionable whether complete stability can be attained in their modified testpiece since the gsf is not significantly improved. However, they claim that crack jumps are minimized [6]. In a previous paper by one of us [2], it was shown that stable quasi-static cracking in an epoxy resin can be achieved by putting adhesive tapes across the crack paths in beam-like specimens, which act as effective elastic springs.

In addition, it was shown that stable cracking in epoxy resins could also be achieved by using a composite testpiece incorporating a $\mathrm{d} R / \mathrm{d} \dot{a}$ positive material which also cracked in parallel with the epoxy. This is done by sticking the two testpieces together with a single crack penetrating both. Fig. 1 shows an example where an epoxy resin sheet was stabilized by a Perspex sheet in parallel. The effective toughness of the composite testpiece is given by

$$
R=\frac{R_{\mathrm{p}} t_{\mathrm{p}}+R_{\mathrm{e}} t_{\mathrm{e}}}{\left(t_{\mathrm{p}}+t_{\mathrm{e}}\right)}
$$

where $R_{\mathrm{p}}, R_{\mathrm{e}}$ are fracture toughness values for Perspex plate and the epoxy resin respectively and $t_{\mathrm{p}}, t_{\mathrm{e}}$ the corresponding thicknesses. When $R_{\mathrm{p}}, t_{\mathrm{p}}$ and $t_{\mathrm{e}}$ are known, $R_{\mathrm{e}}$ can be easily calculated. Such fracture toughness figures for the epoxy resin are shown in the figure.

Therefore, by suitable experimentation, it is possible to derive the $R-\dot{a}$ relation for epoxy resins with no "crack jumps". Fig. 2 shows the variation of $R$ with $\dot{a}$ for an epoxy resin which is $6.5 \mathrm{~mm}$ thick [7]. It may be seen that $\mathrm{d} R / \mathrm{d} \dot{a}$ is negative and substantiates the results obtained in [1]. Furthermore, $d R / \mathrm{d} \dot{a}$ is very negative for slow crack speeds.

From these experimental results, it is obvious that it is possible to obtain stable cracking (called "tearing" by the authors) without jumps in epoxy resins. Comparison of fracture surface energies or fracture toughness values based on appropriate crack propagation speeds can then be easily made.

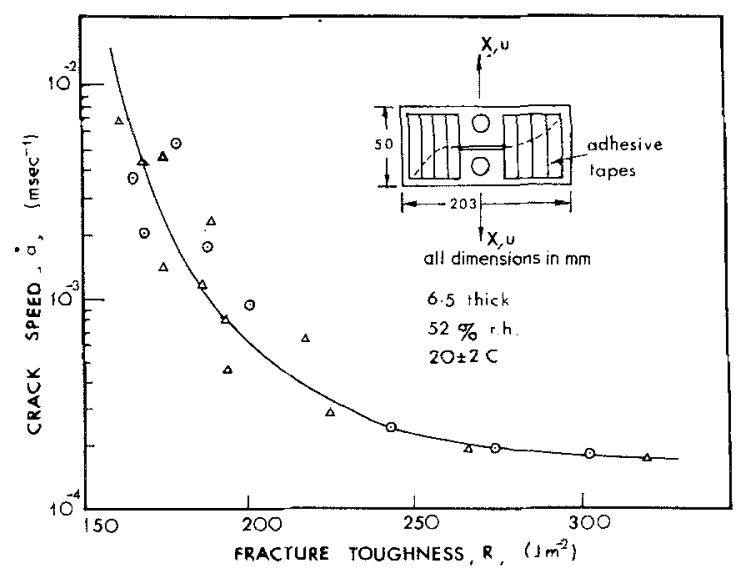

Figure 2 Variation of $R$ with $\dot{a}$ for an epoxy resin. 


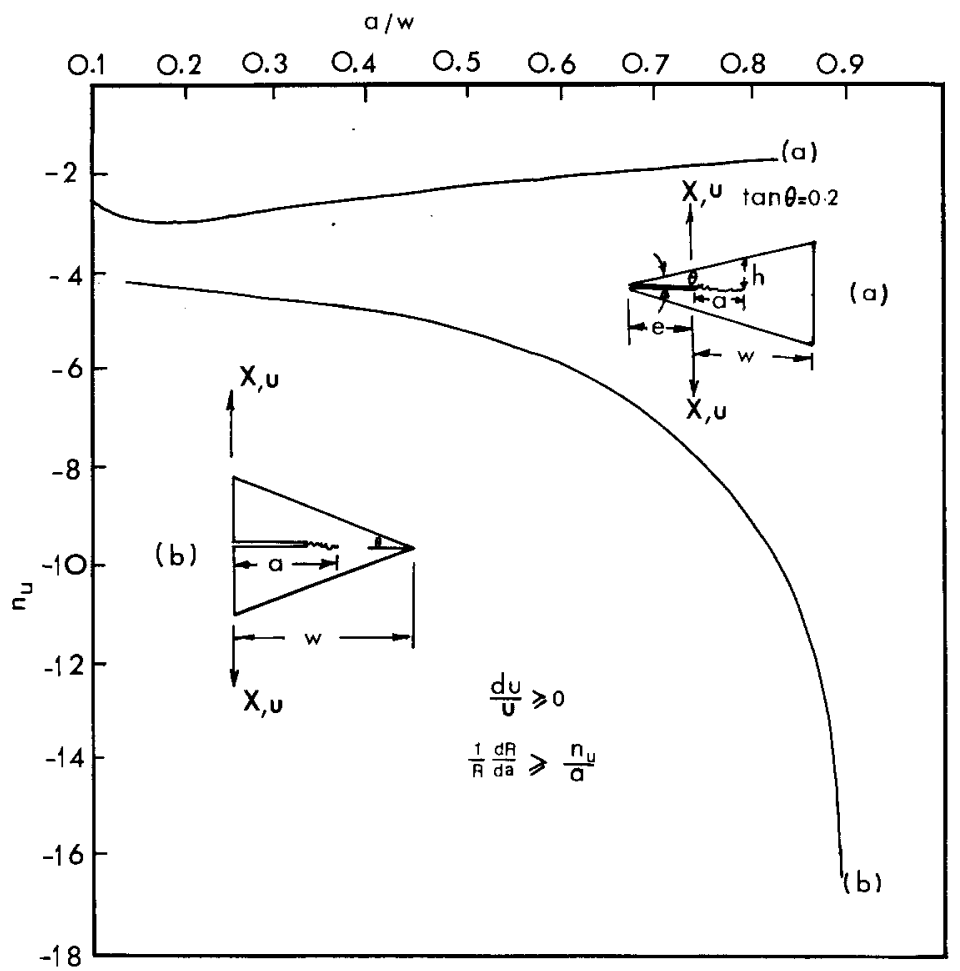

Figure 3 Stability factors in a stiff testing machine for conventionally and modified loaded TDCB specimens.
In this respect, the usefulness of the Gurney method can be extended to such materials, and then consistent toughness results should be obtained by any of the three methods used by Selby and Miller.

When instabilities occur, and crack arrest "saw tooth" load/extension plots are obtained, the $R$ value obtained by Gurney's method is related to the $R$ value at initiation and the $R$ value at arrest (determined by Berry's method or the Irwin-Kies equation) by [10]

$$
R_{\mathrm{G}}=\sqrt{ }\left(R_{\mathrm{I}} R_{\mathrm{A}}\right)
$$

where $R_{\mathrm{G}}$ is the Gurney answer. Clearly $R_{\mathrm{A}}<$ $R_{\mathrm{G}}<R_{\mathrm{I}}$, and $R_{\mathrm{G}}$ represents some average dynamic toughness at an unknown average velocity during the "crack jump". If $R(\dot{a})$ information (such as in Fig. 2) were known for the authors' material, some estimate of that crack speed could be obtained.

The question of crack stability may be even more important in tests conducted in hostile environments. For instance, it has been shown the effects of absorption of water at crack tips can also cause crack jumps $[5,7]$ during fracture 2002 toughness testing. Apparently, this phenomenon of unstable cracking can be explained in terms of an increase in toughness of the local material at the crack tip due to absorption of water; then as the crack passes through the water-affected zone into the virgin material which has negligible absorption (and hence a lower toughness value), $\mathrm{d} R / \mathrm{d} a$ becomes so much negative that the stability criteria as given in Equations 1 and 2 are not satisfied.

The final point we would like to raise concerns the widespread use of TDCB specimens to measure fracture surface energies. We have recently shown $[8,9]$ that, apart from its useful constant rate of change of compliance characteristic, the TDCB specimen is a worse test geometry than the DCB with parallel arms as far as crack stability is concerned. Increasing the taper angle of the testpiece worsens the stability of cracking. However, by turning the conventional TDCB specimen around with loadings applied at the thick base $[8,9]$ crack stability is markedly increased. Fig. 3 compares the variation of $n_{u}$ with $a / w$ for TDCB testpieces when loadings were applied in the manner shown in the irset. 


\section{References}

1. K. SELBY and L. E. MILleR, J. Mater. Sci. 10 (1975) 12.

2. C. GURNEY and Y. W. MAI, Eng. Fract. Mech. 4 (1972) 853.

3. C. GURNEY and J. HUNT, Proc. Roy. Soc. A299 (1967) 508.

4. C. GURNEY and K. M. NGAN, ibid A325 (1972) 207.

5. A. D. S. DIGWAA, Polymer 15 (1974) 101.

6. W. T. EVANS and B. I. G. BARR, J. Strain Analysis 9 (1974) 166.

7. Y. W. MAI, unpublished research, University of Hong Kong (1972).
8. Y. W. MAI, A. G. ATKINS and R. M. CADDELL, Int. J. Fracture (1975) in press.

9. Y. W. MAI, ibid 10 (1974) 292.

10. R. G. HOAGLAND and A. R. ROSENFIELD, ibid 10 (1974) 299.

\section{Received 21 March}

and accepted 14 April 1975

Y.W. MAI

A. G. ATKINS ${ }^{*}$

Department of Mechanical Engineering, University of Michigan, Ann Arbor, Michigan,

USA

*Present address: Delta Materials Research Lid, Box 22, Hadleigh Road, Ipswich, Suffolk, UK.

Reply to comments on "The velocity dependent fracture toughness of epoxy resins"

In reply to Drs Mai and Atkins' comments concerning the velocity dependence of fracture toughness in epoxy resins, we should like to say that we are grateful for their clarification of the stability aspects of the TDCB specimen. We feel that the analysis provided by them endorses our original view that one should exercise caution when faced with a combination of material and test geometry which involves the "crack-jumping" situation. As they quite rightly point out, knowledge of the rate sensitivity of the fracture process is of paramount importance. In the context of our original investigation, however, the fracture toughness measurement was intended as much as a monitoring device for morphological changes in the resin as it was for generating engineering data. It was found that the TDCB specimen provided a reliable source of information on both these aspects and indeed, its very susceptibility to unstable cracking was instrumental in suggesting other investigations concerning the variation of mechanical properties with changes in stoichiometry.

Received and accepted 19 May 1975

K. SELBY

Department of Materials Technology, Loughborough University of Technology, Loughborough, UK L. E. MILLER Redland Technology Ltd, Graylands, Horsham, Sussex, UK

\section{Observation of processes of superplasticity with the scanning electron microscope}

Tensile tests have been performed in the scanning electron microscope on a eutectic $\mathrm{Pb}-\mathrm{Sn}$ alloy having super-plastic properties. In this way it was possible to follow the dynamics of the events during deformation at a microscopic level. The specimens were electrolitically attacked in order to remove the superficial layer and to expose single grains. Marker scratches were made on the surface with a $0.5 \mu \mathrm{m}$ diamond paste. The difference in atomic number between $\mathrm{Pb}$ and $\mathrm{Sn}$ allowed one to distinguish between grains of different com(c) 1975 Chapman and Hall Ltd. Printed in Great Britain. position, especially when using back-scattered electrons.

From the figures some conclusions can be drawn about the mechanisms acting during the deformation of this superplastic alloy. The grains which originally formed the surface remain at the surface during the entire deformation (grains denoted by $0,1 \ldots \mathrm{g}, \mathrm{a}, \mathrm{h}, \mathrm{m}, \mathrm{n}$, in Fig. 1). They do not change their form to a measurable extent. During the initial period of deformation they are shifted against one another but are later separated by new grains coming to the surface (Fig. 1d). This is only possible if the grains slide against one another. Our observations are limited to the 